\title{
miR-16 promotes the apoptosis of human cancer cells by targeting FEAT
}

Hongwei Liang ${ }^{2+}$, Zheng Fu ${ }^{2+}$, Xueyuan Jiang ${ }^{2,4+}$, Nan Wang ${ }^{2}$, Feng Wang ${ }^{5}$, Xueliang Wang ${ }^{2}$, Suyang Zhang ${ }^{2}$, Yanbo Wang ${ }^{2}$, Xin Yan ${ }^{3}$, Wen-xian Guan ${ }^{5}$, Chen-Yu Zhang ${ }^{2}$, Ke Zen ${ }^{2}$, Yujing Zhang ${ }^{2^{*}}$, Xi Chen $^{2^{*}}$ and Guangxin Zhou ${ }^{1^{*}}$

\begin{abstract}
Background: Although human cancers have heterogeneous combinations of altered oncogenes, some crucial genes are universally dysregulated in most cancers. One such gene, FEAT (faint expression in normal tissues, aberrant overexpression in tumors), is uniformly overexpressed in a variety of human cancers and plays an important role in tumorigenesis by suppressing apoptosis. However, the precise molecular mechanism through which FEAT is upregulated during tumorigenesis remains largely unknown.

Methods: In this study, we used bioinformatic analyses to search for miRNAs that potentially target FEAT. We examined the expression of FEAT protein level by western blotting and miR-16 level by qRT-PCR assay. Cancer cell lines (A549, MCF-7 and Huh-7) with miR-16 upregulation and FEAT silencing were established and the effects on apoptosis of cancer cells in vitro were assessed. Luciferase reporter assay was also performed to investigate the interaction between miR-16 and FEAT.

Results: We identified a specific target site for miR-16 in the 3'-untranslated region (3'-UTR) of FEAT. Consistent with the bioinformatic analyses, we identified an inverse correlation between the miR-16 and FEAT protein levels in lung cancer, breast cancer, and hepatocellular cancer tissues. We then experimentally validated miR-16 as a direct regulator of FEAT using cell transfection and luciferase assays. Finally, we demonstrated that the repression of FEAT by miR-16 promoted the apoptosis of cancer cells.
\end{abstract}

Conclusions: Our findings provide the first clues regarding the role of miR-16 as a tumor suppressor in cancer cells through the inhibition of FEAT translation.

Keywords: miR-16, FEAT, Apoptosis

\section{Background}

Although our understanding of the molecular mechanisms of carcinogenesis has greatly improved, this knowledge has not led to the identification and development of effective tools for cancer screening and prevention. In theory, one of the most feasible and promising approaches for cancer screening and prevention is targeting a common oncogene that occurs in most tumors. However, the marked heterogeneity and complexity of human tumors make it difficult to identify commonalities among cancers

\footnotetext{
* Correspondence: yjzhang@nju.edu.cn; xichen@nju.edu.cn; oxis@163.com ${ }^{\dagger}$ Equal contributors

${ }^{2}$ Jiangsu Engineering Research Center for microRNA Biology and Biotechnology, State Key Laboratory of Pharmaceutical Biotechnology, School of Life Sciences, Nanjing University, 22 Hankou Road, Nanjing, Jiangsu 210093, China

${ }^{1}$ Department of Orthopedics, Jinling Hospital, School of Medicine, Nanjing University, 305 East Zhongshan Road, Nanjing, Jiangsu 210002, China

Full list of author information is available at the end of the article
}

$[1,2]$. Oncogenes that contribute to the development of human cancers are highly variable among different types of cancer and among individual tumors of the same type $[1,2]$. Thus, it is still poorly understood whether there are crucial oncogenes that are commonly altered in diverse cancers. Recently, Takahashi et al. investigated a previously unrecognized protein, FEAT (faint expression in normal tissues, aberrant overexpression in tumors), and identified it as a novel, prominent promoter of tumorigenesis [3]. FEAT protein is encoded by METTL13 gene (methyltransferase like 13), and it is aberrantly overexpressed in most human cancers but weakly expressed in normal tissues [3]. Remarkably, transgenic mice that ectopically expressed FEAT spontaneously developed tumors, indicating that the FEAT protein potently drives tumorigenesis in vivo [3]. Gene expression profiling has suggested that FEAT drives receptor tyrosine kinase and hedgehog signaling pathways

\section{Biomed Central}

(c) 2015 Liang et al.; licensee BioMed Central. This is an Open Access article distributed under the terms of the Creative Commons Attribution License (http://creativecommons.org/licenses/by/4.0), which permits unrestricted use, distribution, and reproduction in any medium, provided the original work is properly credited. The Creative Commons Public Domain Dedication waiver (http://creativecommons.org/publicdomain/zero/1.0/) applies to the data made available in this article, unless otherwise stated. 
[3]. However, despite these recent advances in our understanding of the important roles of FEAT in cancer progression, the precise molecular mechanism through which FEAT is upregulated during tumorigenesis remains largely unknown. Further studies are needed to fully elucidate the regulation of FEAT expression in normal and neoplastic tissues.

microRNAs (miRNAs) are a class of endogenously expressed, small non-coding RNAs that play an important role in the regulation of gene expression at the posttranscriptional level [4-6]. Some of these miRNAs have attracted special attention for their involvement in the initiation, progression, and metastasis of human cancers $[7,8]$. One particularly well-studied example is the ubiquitously expressed and highly conserved miR-16, one of the first miRNAs that was known to be linked to human malignancies [9]. Evidence indicates that miR-16 can modulate the cell cycle, inhibit cell proliferation, promote cell apoptosis, and suppress tumorigenicity both in vitro and in vivo [10]. These effects can be explained by several targets of miR-16: the anti-apoptotic gene B-cell lymphoma 2 (Bcl-2) [11]; numerous genes involved in the G1-S transition such as CCND1 (cyclin D1), CCND3 (cyclin D3), CCNE1 (cyclin E1), and CDK6 (cyclin-dependent kinase 6) [12-14]; and genes involved in the Wnt signaling pathway, such as WNT3A (wingless-type MMTV integration site family, member 3A) [14]. Consistently, miR-16 is frequently deleted and/or downregulated in many types of cancer, including chronic lymphocytic leukemia $[9,15]$, prostate cancer [14], and lung cancer [16]. Thus, miR-16 is generally thought to be a key tumor-suppressive miRNA that can target numerous oncogenes in various human cancers.

Although the dysregulation of miR-16 and FEAT plays an important role in carcinogenesis, no correlation between FEAT and miR-16 in cancers has been reported. In this study, we hypothesized that FEAT is a target of miR-16. After measuring the expression levels of miR-16 and FEAT in different types of human cancer tissues and paired noncancerous tissues, we detected an inverse correlation between miR-16 and FEAT in human cancers. Furthermore, in this study, we experimentally investigated the direct regulation of FEAT by miR-16 and the biological role of miR-16 targeting FEAT in human cancer cells.

\section{Methods}

\section{Cells and human tissues}

The human lung cancer cell lines A549, human breast cancer cell lines MCF-7, and human liver cancer cell lines Huh-7 were purchased from the Shanghai Institute of Cell Biology, Chinese Academy of Sciences (Shanghai, China). A549, MCF-7, and Huh-7 cells were cultured in DMEM supplemented with $10 \%$ fetal bovine serum (GIBCO, CA, USA). All cells were incubated in a $5 \% \mathrm{CO}_{2}$ at $37{ }^{\circ} \mathrm{C}$ in a watersaturated atmosphere. The tumors and paired normal adjacent tissues were derived from patients undergoing a surgical procedure at the Affiliated Gulou Hospital of Nanjing University (Nanjing, China). All of the patients or their guardians provided written consent, and the Ethics Committee from Nanjing University approved all aspects of this study. Tissue fragments were immediately frozen in liquid nitrogen at the time of surgery and stored at $-80{ }^{\circ} \mathrm{C}$. The clinical features of the patients are listed in Table 1.

\section{RNA isolation and quantitative RT-PCR}

Total RNA was extracted from the cultured cells and human tissues using TRIzol Reagent (Invitrogen, Carlsbad, CA) according to the manufacturer's instructions. Assays to quantify miRNAs were performed using Taqman miRNA probes (Applied Biosystems, Foster City, CA) according to the manufacturer's instructions. Briefly, $1 \mu \mathrm{g}$ of total RNA was reverse-transcribed to cDNA using AMV reverse transcriptase (TaKaRa, Dalian, China) and a stem-loop RT primer (Applied Biosystems). The reaction conditions were as follows: $16{ }^{\circ} \mathrm{C}$ for $30 \mathrm{~min}, 42{ }^{\circ} \mathrm{C}$ for $30 \mathrm{~min}$, and $85{ }^{\circ} \mathrm{C}$ for 5 min. Real-time PCR was performed using a TaqMan PCR kit on an Applied Biosystems 7300 Sequence Detection System (Applied Biosystems). The reactions were incubated in a 96-well optical plate at $95^{\circ} \mathrm{C}$ for $10 \mathrm{~min}$, followed by $40 \mathrm{cy}$ cles of $95^{\circ} \mathrm{C}$ for $15 \mathrm{~s}$ and $60^{\circ} \mathrm{C}$ for $1 \mathrm{~min}$. All of the reactions were run in triplicate. After the reaction, the cycle threshold $\left(\mathrm{C}_{\mathrm{T}}\right)$ data were determined using fixed threshold settings, and the mean $\mathrm{C}_{\mathrm{T}}$ of the triplicate PCRs was determined. A comparative $\mathrm{C}_{\mathrm{T}}$ method was used to compare each condition with the controls. The relative levels of the miRNAs in the cells and tissues were normalized to U6. The amount of miRNA relative to the internal control U6 was calculated using the $2^{-\Delta \Delta C T}$ equation, in which $\Delta \Delta C_{T}=\left(C_{T}\right.$ miRNA $\left.\mathrm{C}_{\mathrm{T} \text { U6 }}\right)_{\text {target }}-\left(\mathrm{C}_{\mathrm{T} \text { miRNA }}-\mathrm{C}_{\mathrm{T}} \mathrm{U} 6\right)_{\text {control. }}$. To quantify the FEAT mRNA, $1 \mu \mathrm{g}$ of total RNA was reverse-transcribed to cDNA using oligo $(\mathrm{dT})$ and Thermoscript (TaKaRa) in the reaction, which was performed under the following conditions: $42{ }^{\circ} \mathrm{C}$ for $60 \mathrm{~min}$ and $70{ }^{\circ} \mathrm{C}$ for $10 \mathrm{~min}$. Next, real-time PCR was performed using the RT product, SYBER Green Dye (Invitrogen) and specific primers for FEAT and GAPDH. The sequences of the primers were as follows: FEAT (sense): 5'-CTTCACCGAGGTCAGCAGTA-3'; FEAT (antisense): 5'-CTCCATGACTCTAGCCGACA-3'; GAPDH (sense): 5'-GATATTGTTGCCATCAATGAC-3'; and GAPDH (antisense): 5'-TTGATTTTGGAGGGATCTCG-3'. The reactions were incubated at $95{ }^{\circ} \mathrm{C}$ for $5 \mathrm{~min}$, followed by 40 cycles of $95{ }^{\circ} \mathrm{C}$ for $30 \mathrm{~s}, 60^{\circ} \mathrm{C}$ for $30 \mathrm{~s}$, and $72{ }^{\circ} \mathrm{C}$ for $30 \mathrm{~s}$. After the reactions were complete, the $\mathrm{C}_{\mathrm{T}}$ values were determined by setting a fixed threshold. The relative amount of FEAT mRNA was normalized to GAPDH.

\section{Overexpression and knockdown of miR-16}

Synthetic pre-mir-16, anti-mir-16, and scrambled negative control RNAs were purchased from Ambion (Austin, TX, 
Table 1 Clinical Features of cancer patients

\begin{tabular}{lll}
\hline Cancer type & Tumor subtype & Pathological stage \\
\hline Lung cancer \#1 & Adenocarcinoma & IIIA \\
Lung cancer \#2 & Adenocarcinoma & IIB \\
Lung cancer \#3 & Squamous cell carcinoma & IIIA \\
Breast cancer \#1 & Invasive Ductal Carcinoma & III \\
Breast cancer \#2 & Invasive Ductal Carcinoma & III \\
Breast cancer \#3 & Invasive Ductal Carcinoma & $\|$ \\
Hepatocellular cancer \#1 & Hepatocellular carcinoma & $\|$ \\
Hepatocellular cancer \#2 & Hepatocellular carcinoma & $\|$ \\
Hepatocellular cancer \#3 & Hepatocellular carcinoma & III \\
\hline
\end{tabular}

USA). All cells were seeded in 6-well plates or $60-\mathrm{mm}$ dishes, and the cells were transfected with Lipofectamine 2000 (Invitrogen) on the following day, when the cells were approximately $70 \%$ confluent. In each well, equal amounts of pre-mir-16, anti-mir-16, or scrambled negative control RNA were used. The cells were harvested $24 \mathrm{~h}$ after transfection for quantitative RT-PCR and Western blotting.

\section{Luciferase reporter assay}

To test the direct binding of miR-16 to the target gene FEAT, a luciferase reporter assay was performed as previously described [17]. The entire 3 '-untranslated region (3'-UTR) of human FEAT was amplified using PCR with human genomic DNA as a template. The PCR products were inserted into the p-MIR-reporter plasmid (Ambion), and the insertion was confirmed to be correct via sequencing. To test the binding specificity, the sequences that interacted with the miR-16 seed sequence were mutated (from UGCUGCU to ACGACGA), and the mutant FEAT 3 '-UTR was inserted into an equivalent luciferase reporter. For luciferase reporter assays, A549, MCF-7, and Huh-7 cells were cultured in 24-well plates, and each well was transfected with $1 \mu \mathrm{g}$ of firefly luciferase reporter plasmid, $1 \mu \mathrm{g}$ of a $\beta$-galactosidase $(\beta$-gal) expression plasmid (Ambion), and equal amounts (100 pmol) of pre-mir16 , anti-mir-16, or the scrambled negative control RNA using Lipofectamine 2000 (Invitrogen). The $\beta$-gal plasmid was used as a transfection control. Twenty-four hours post-transfection, the cells were assayed using a luciferase assay kit (Promega, Madison, WI, USA).

\section{Plasmid construction and siRNA interference assay}

An siRNA sequence targeting the human FEAT cDNA was purchased from Santa Cruz (sc-88139, Santa Cruz Biotechnology, CA, USA). A scrambled siRNA was included as a negative control. A mammalian expression plasmid encoding the human FEAT open reading frame (pReceiver-M02-FEAT) was purchased from GeneCopoeia (Germantown, MD, USA). An empty plasmid served as a negative control. The FEAT expression plasmid and FEAT
siRNA were transfected into A549 cells using Lipofectamine 2000 (Invitrogen) according to the manufacturer's instructions. Both total RNA and protein were isolated $24 \mathrm{~h}$ posttransfection. The FEAT mRNA and protein expression levels were assessed by quantitative RT-PCR and Western blotting.

\section{Protein extraction and western blotting}

All cells were rinsed with PBS ( $\mathrm{pH} 7.4)$ and lysed in RIPA Lysis buffer (Beyotime, China) supplemented with a Protease and Phosphatase Inhibitor Cocktail (Thermo Scientific 78440) on ice for $30 \mathrm{~min}$. The tissue samples were frozen solid with liquid nitrogen, ground into a powder and lysed in RIPA Lysis buffer containing the Protease and Phosphatase Inhibitor Cocktail on ice for $30 \mathrm{~min}$. When necessary, sonication was used to facilitate lysis. Cell lysates or tissue homogenates were centrifuged for $10 \mathrm{~min}\left(12000 \mathrm{~g}, 4^{\circ} \mathrm{C}\right)$. The supernatant was collected, and the protein concentration was calculated using a Pierce BCA protein assay kit (Thermo Scientific, Rockford, IL, USA). The protein levels were analyzed using Western blots with the corresponding antibodies. The protein levels were normalized by probing the same blots with a GAPDH antibody. The antibodies were purchased from the following sources: anti-FEAT (X-20) (sc-101995, Santa Cruz Biotechnology, CA, USA) and antiGAPDH (sc-365062, Santa Cruz Biotechnology, CA, USA). Protein bands were analyzed using the ImageJ software.

\section{Apoptosis assays}

The apoptosis of A549 cells was tested using an Annexin V-FITC/propidium iodide (PI) staining assay. A549 cells were cultured in 12-well plates and transfected with premir-16, anti-mir-16, FEAT siRNA, or the FEAT overexpression plasmid to induce apoptosis. Pre-mir-control, anti-mir-control, control siRNA, and control plasmid served as negative controls. Cells were cultured overnight with both serum-containing complete medium and serum-depleted medium; and the attached and floating cells were then harvested. Flow cytometry analysis of apoptotic cells was performed using an Annexin V-FITC/PI staining kit (BD Biosciences, CA, USA). After washes with 
cold PBS, the cells were resuspended in binding buffer (100 mM HEPES, pH 7.4, $100 \mathrm{mM} \mathrm{NaCl}$, and $25 \mathrm{mM}$ $\mathrm{CaCl}_{2}$ ), followed by staining with Annexin V-FITC/PI at room temperature in darkness for $15 \mathrm{~min}$. Apoptotic cells were then evaluated by gating PI and Annexin V-positive cells on a fluorescence-activated cell-sorting (FACS) flow cytometer (BD Biosciences, San Jose, CA). All experiments were performed in triplicate.

\section{Functional annotation}

Based on miRTarBase, there are now more than 100 experimental validated target genes of miR-16 [18]. the Gene Ontology (GO) classification was performed to gain insights into the biological functions of miR-16 target genes, and the Kyoto Encyclopedia of Genes and Genomes (KEGG) pathway enrichment analysis was performed to detect the potential pathway of miRNA target genes. KEGG pathway database is a recognized and comprehensive database including all kinds of biochemistry pathways [19]. The online based software GENECODIS was utilized in those functional annotation [20].

\section{Statistical analysis}

All of the Western blotting images are representative of at least three independent experiments. Quantitative RT-PCR, the luciferase reporter assay, and the cell viability and apoptosis assays were performed in triplicate, and each experiment was repeated several times. The data shown are the mean $\pm S E$ of at least three independent experiments. The differences were considered statistically significant at $\mathrm{p}<0.05$ using Student's $t$-test.

\section{Results}

Upregulation of FEAT protein in human cancer tissues

We first determined the expression patterns of FEAT in lung cancer, breast cancer, and hepatocellular cancer tissues. After measuring the protein levels of FEAT in these cancer tissues and the corresponding noncancerous tissues, we found that FEAT protein is dramatically overexpressed in cancer tissues but totally absent in normal tissues (Fig. 1a). However, FEAT mRNA was readily detected in noncancerous tissues, and its levels were slightly upregulated in cancer tissues (Fig. 1b). This disparity between protein and mRNA in FEAT expression in cancers strongly suggests that a post-transcriptional mechanism is involved in FEAT regulation.

\section{Identification of conserved miR-16 target sites within the 3'-UTR of FEAT}

One important mode of post-transcriptional regulation is the repression of mRNA transcripts by miRNAs. miRNAs are therefore likely to play a biologically relevant role in regulating FEAT expression in cancer. Three computational algorithms, including TargetScan [21], miRanda
[22], and PicTar [23], were used in combination to identify potential miRNAs that can target FEAT. Using these approaches, miR-16 was identified as a candidate regulatory miRNA of FEAT. The predicted interaction between miR-16 and the target sites in the FEAT 3'-UTR are illustrated in Fig. 1c. One potential miR-16 target site was found in the 3'-UTR of the FEAT mRNA sequence. The minimum free energy value of this hybrid is $-22.0 \mathrm{kcal} / \mathrm{mol}$, which is well within the range of genuine miRNA-target pairs. Moreover, perfect base pairing occurs between the seed region (the core sequence that encompasses the first 2-8 bases of the mature miRNA) and the cognate targets. Furthermore, the miR-16 binding sequence in the FEAT 3 '-UTR is highly conserved across species.

\section{Detection of an inverse correlation between the miR-16 and FEAT levels in cancer tissues}

miRNAs are generally thought to have expression patterns that are the opposite of those of their targets [4-6]. We next investigated whether miR-16 was inversely correlated with FEAT in cancer tissues. After determining the levels of miR-16 in the same three pairs of lung cancer, breast cancer, and hepatocellular cancer tissues and the corresponding noncancerous tissues, we showed that the miR-16 levels were consistently downregulated in cancer tissues (Fig. 1d). Combining the computational prediction with the detection of inverse correlation between miR-16 and FEAT in vivo, it is quite likely that miR-16 is involved in the post-transcriptional regulation of FEAT.

\section{Validation of FEAT as a direct target of miR-16}

The correlation between miR-16 and FEAT was further examined by evaluating FEAT expression in human lung adenocarcinoma A549 cells, human breast adenocarcinoma MCF-7 cells, and human hepatocellular carcinoma Huh-7 cells after overexpressing or knocking down miR-16. In these experiments, miR-16 overexpression was achieved by transfecting the cells with pre-mir-16 (a synthetic RNA oligonucleotide duplex mimicking the miR-16 precursor), and miR-16 knockdown was achieved by transfecting the cells with anti-mir-16 (a chemically modified antisense oligonucleotide designed to specifically target mature miR-16). As anticipated, the miR-16 levels were significantly increased in A549, MCF-7, and Huh-7 cells when these cells were transfected with pre-mir-16, whereas the miR-16 levels were decreased when these cells were transfected with anti-mir-16 (Fig. 2a). The expression of the FEAT protein was reduced by the overexpression of miR-16 and increased by the knockdown of miR-16 in A549, MCF-7, and Huh-7 cells (Fig. 2, b and c). To determine the level at which miR-16 influenced FEAT expression, we repeated the above-mentioned experiments and examined the expression of FEAT mRNA after transfection. Although the intracellular level of miR-16 was 


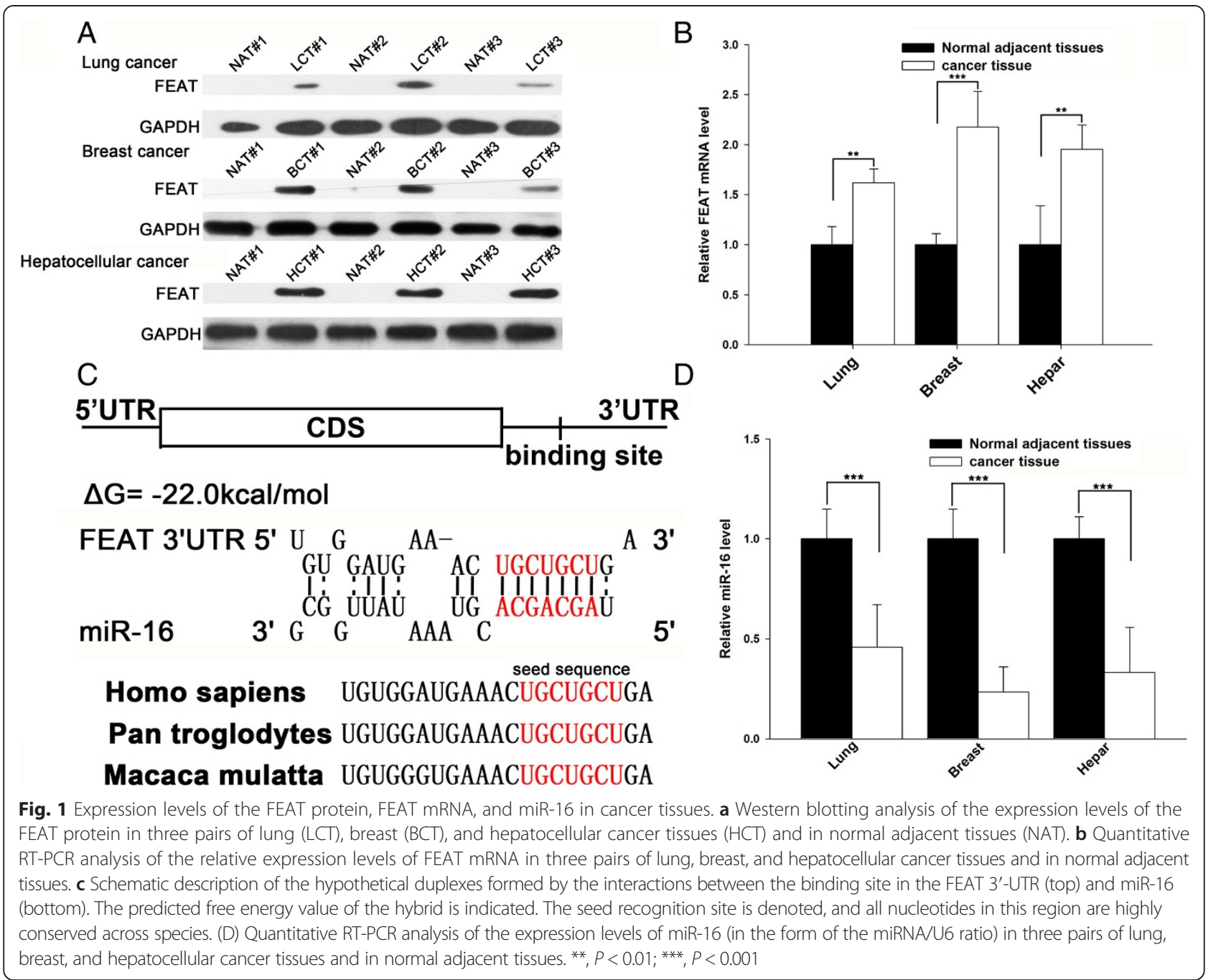

significantly altered after transfection with pre-mir-16 and anti-mir-16, the alteration of the miR-16 levels did not affect the FEAT mRNA levels (Fig. 2d). These results demonstrate that miR-16 specifically regulates FEAT protein expression at the post-transcriptional level, which is a typical miRNA-mediated regulation mechanism.

To determine whether the negative regulatory effects that miR-16 exerted on FEAT expression were mediated through the binding of miR-16 to the presumed sites in the 3 '-UTR of the FEAT mRNA, the full-length FEAT 3 '-UTR that contained the sole presumed miR-16 binding site was fused downstream of the firefly luciferase gene in a reporter plasmid. The resulting plasmid was transfected into A549, MCF-7, and Huh-7 cells along with pre-mir-16 or anti-mir-16. As expected, the overexpression of miR-16 resulted in a significant reduction of luciferase reporter activity compared with transfection with pre-scramble control, whereas the inhibition of miR-16 resulted in an increase in reporter activity compared with transfection with anti-scramble control (Fig. 2e). Furthermore, we introduced point mutations into the corresponding complementary sites in the FEAT 3'-UTR to eliminate the predicted miR-16 binding site. This mutated luciferase reporter was unaffected by both the overexpression and knockdown of miR-16 (Fig. 2e). This finding suggests that the binding site strongly contributes to the interaction between miR-16 and FEAT mRNA. In conclusion, our results demonstrate that miR-16 directly recognizes and binds to the 3'-UTR of the FEAT mRNA transcript thereby inhibiting FEAT translation.

miR-16 promotes the apoptosis of cancer cells by regulating $\mathrm{FEAT}$

We next focused on studying the role of miR-16 in regulating FEAT. Because FEAT is known to be involved in cell apoptosis regulation [3], we investigated whether the overexpression or knockdown of miR-16 or FEAT would impact cell apoptosis in A549 cells using flow cytometry analysis. The efficient overexpression or knockdown of FEAT is shown in Fig. 3, a-c. In support of the notion 


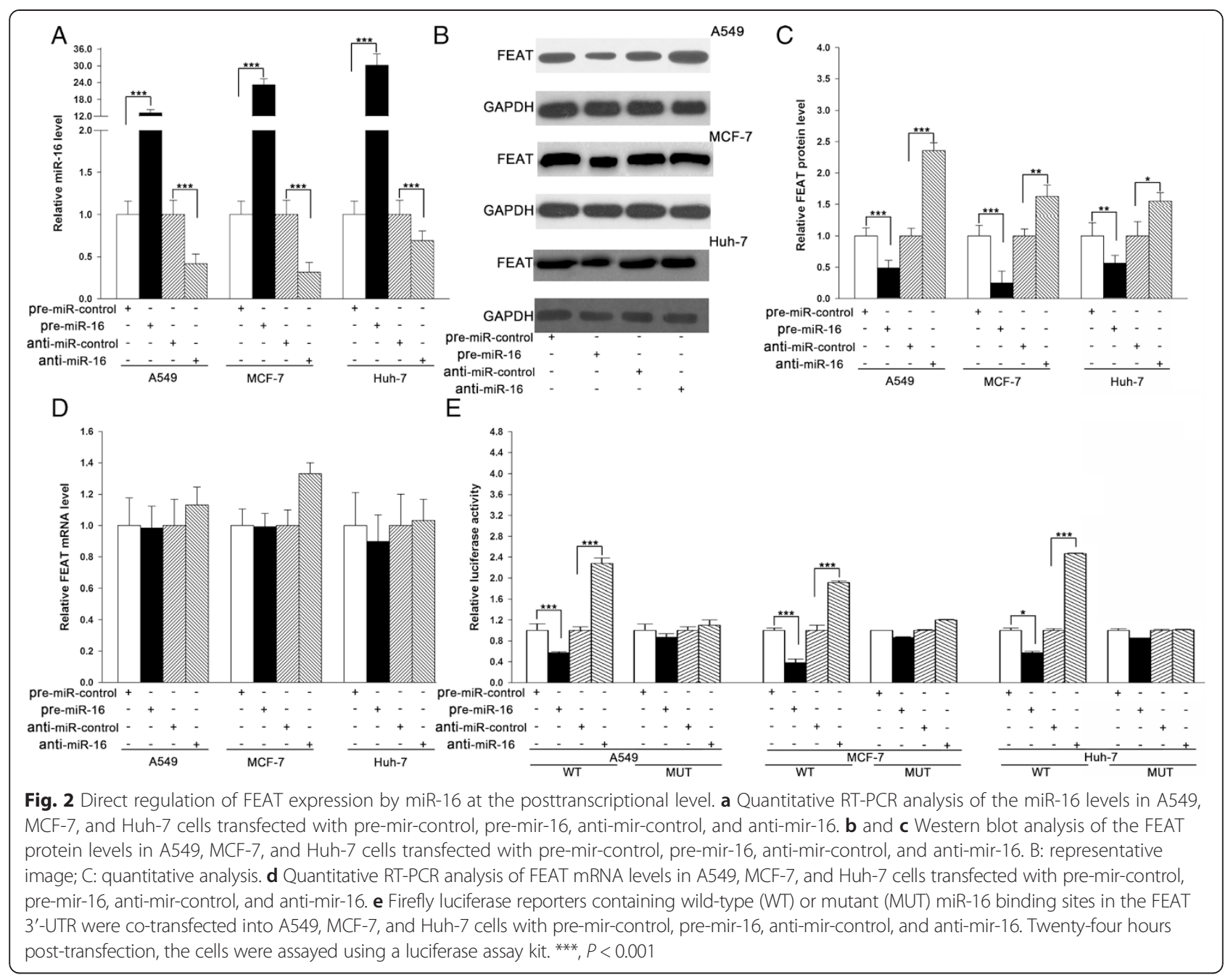

that FEAT is essential in suppressing apoptosis [3], A549 cells transfected with FEAT siRNA showed a promotion of cell apoptosis (Fig. 3, d and e). In contrast, transfection with the FEAT-overexpressing plasmid, which specially expresses the full-length open reading frame (ORF) of FEAT without the miR-16-responsive 3'-UTR, had an opposite effect on cell apoptosis (Fig. 3, d and e). Subsequently, we assessed the role of miR-16 in cell apoptosis. As expected, A549 cells transfected with pre-mir-16 exhibited a significantly higher rate of cell apoptosis, whereas A549 cells transfected with anti-mir-16 had a lower apoptosis rate (Fig. 3, d and e). Moreover, compared with cells that had been transfected with pre-mir-16, those transfected with pre-mir-16 and the FEAT-overexpressing plasmid exhibited significantly lower apoptosis rates (Fig. 3, d and e), suggesting that miR-16-resistant FEAT is sufficient to rescue the suppression of FEAT by miR-16 and attenuate the pro-apoptotic effect of miR-16 on cancer cells. Taken together, the results indicate that miR-16 can promote cell apoptosis by silencing FEAT.

\section{Discussion}

The significance of overexpressed proteins in cancer is recognized as a potential lead for a variety of diagnostic and therapeutic approaches for cancers. Studies that identify and characterize common oncogenic proteins will hopefully advance molecular-targeted cancer screening and prevention. However, due to the marked heterogeneity and complexity of different types of human cancers, it is rather difficult to identify such proteins that are commonly altered in diverse cancers. Notably, a previously unrecognized protein, FEAT, was recently found to be highly expressed in an unusually wide range of tumors but not expressed in most normal tissues [3], suggesting that FEAT is a ubiquitous protein that is involved in human cancer. FEAT was originally purified from rat livers as a protein that inhibits nuclear apoptosis in vitro [3]. Ex vivo experiments confirmed that FEAT attenuates apoptotic cell death [3]. Studies have demonstrated that FEAT is highly oncogenic in vivo [3]. However, despite these recent advances in our understanding of the important roles of FEAT in cancer 


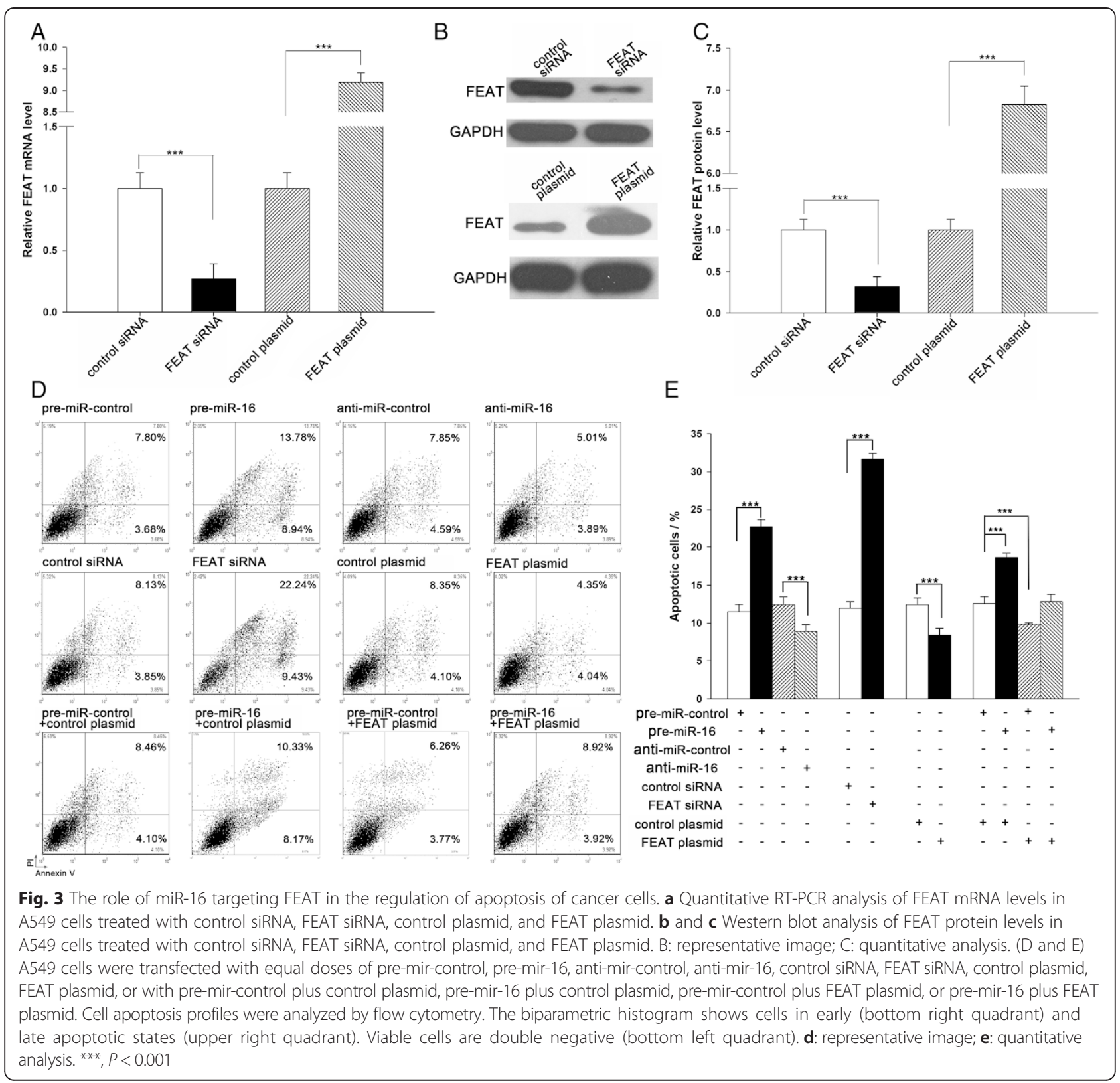

progression, the precise molecular mechanism through which FEAT is upregulated during tumorigenesis remains largely unknown. In this study, we showed that silencing FEAT expression using siRNA could promote cell apoptosis in cancer cells, whereas overexpressing FEAT had an opposite effect, validating its role as an essential oncogenic protein during tumorigenesis. Interestingly, we identified discordance between the FEAT protein and mRNA levels in human lung cancer, breast cancer, and hepatocellular cancer tissues. The results suggest that a post-transcriptional regulation mechanism is involved in FEAT repression. One centrally important mode of posttranscriptional regulation is the repression of mRNA transcripts by miRNAs. Therefore, we searched for miRNAs that could target FEAT and experimentally validated miR-16 as a direct regulator of FEAT. The results identified miR-16 as a novel link between the FEAT regulatory pathway and the pathogenesis of cancer. Considering that miR-16 is highly expressed in normal tissues and frequently deleted and downregulated in many types of cancer tissues, the results also explain, at least in part, why FEAT is aberrantly overexpressed in most human cancers but weakly expressed in normal tissues.

In this study, we further investigated whether the cellular phenotypes especially cell apoptosis were regulated by miR-16 targeting FEAT. We showed that miR-16 
could suppress FEAT expression and, in turn, promote apoptosis in cancer cells. The results reveal a critical role for miR-16 as a tumor suppressor and pro-apoptotic molecule in carcinogenesis through the repression of FEAT translation. In fact, miR-16 has been reported to act as a tumor-suppressive miRNA in many cancer types [9-16], and multiple apoptosis-related genes are targeted by miR-16, including BCL-2, CCND1, CCND3, and CCNE1 $[9,13]$. We performed KEGG pathway analysis and GO annotation analysis on the experimental validated target genes of miR-16, and the results showed that most of these target genes were indeed anti-apoptotic factors (Additional file 1 Table S1 and S2). An emerging common theme is that multiple targets regulated by a single miRNA can act in concert, rather than individually, to regulate the same biological process, such as apoptosis. The coordinated regulation of many targets by a single miRNA may allow for a prompt cellular response to the apoptosis signals. In this study, it is noted that restoring FEAT expression can successfully attenuate the pro-apoptotic effects of miR-16 on cancer cells, although miR-16 has many other targets. The results suggest that targeting FEAT is a major mechanism by which miR-16 exerts its tumor-suppressive and proapoptotic function. Therefore, the modulation of FEAT by miR-16 might explain, at least in part, why the downregulation of miR-16 during carcinogenesis can accelerate cancer progression.

Taken together, this study delineates a novel regulatory network employing miR-16 and FEAT to fine-tune cell apoptosis in lung, breast, and hepatocellular cancer cells. This study may provide a potential novel target for future cancer therapy.

\section{Additional file}

Additional file 1: miR-16 promotes the apoptosis of human cancer cells by targeting FEAT.

\section{Competing interests}

The authors declare that they have no competing interests.

\section{Authors' contributions}

Conception and design: Guangxin Zhou, Xi Chen, Yujing Zhang; Development of methodology: Hongwei Liang, Zheng Fu, Xueyuan Jiang; Acquisition of data: Hongwei Liang, Zheng Fu, Xueyuan Jiang, Nan Wang, Feng Wang, Xueliang Wang, Suyang Zhang, Yanbo Wang, Xin Yan; Writing, reviewing, and/ or revision of the manuscript: Guangxin Zhou, Xi Chen, Yujing Zhang; Ke Zen, Chen-Yu Zhang, Wen-xian Guan; Study supervision: Guangxin Zhou, Xi Chen, Yujing Zhang. All authors read and approved the final manuscript.

\section{Acknowledgements}

This work was supported by grants from the National Natural Science Foundation of China (Nos. 81102039, 81472508, 81401895 and 31271378) and the Natural Science Foundation of Jiangsu Province (Nos. BK2012014 and BK20141376).

\section{Author details}

'Department of Orthopedics, Jinling Hospital, School of Medicine, Nanjing University, 305 East Zhongshan Road, Nanjing, Jiangsu 210002, China. 2 Jiangsu Engineering Research Center for microRNA Biology and Biotechnology, State Key Laboratory of Pharmaceutical Biotechnology, School of Life Sciences, Nanjing University, 22 Hankou Road, Nanjing, Jiangsu 210093, China. ${ }^{3}$ The Comprehensive Cancer Center of Drum Tower Hospital Affiliated to Medical School of Nanjing University \& Clinical Cancer Institute of Nanjing University, Nanjing, Jiangsu 210008, China. ${ }^{4}$ Department of Pathology, Keck School of Medicine, University of Southern California, Los Angeles, CA 90033, USA. ${ }^{5}$ Department of General Surgery of Drum Tower Hospital Affiliated to Medical School of Nanjing University, Nanjing, Jiangsu 210008, China.

Received: 4 August 2014 Accepted: 21 May 2015

Published online: 02 June 2015

\section{References}

1. Stratton MR, Campbell PJ, Futreal PA. The cancer genome. Nature. 2009:458(7239):719-24.

2. Hanahan D, Weinberg RA. Hallmarks of cancer: the next generation. Cell. 2011;144(5):646-74.

3. Takahashi A, Tokita H, Takahashi K, Takeoka T, Murayama K, Tomotsune D, et al. A novel potent tumour promoter aberrantly overexpressed in most human cancers. Sci Rep. 2011;1:15.

4. Bartel DP. MicroRNAs: genomics, biogenesis, mechanism, and function. Cell. 2004;116(2):281-97.

5. Ambros V. The functions of animal microRNAs. Nature. 2004;431(7006):350-5.

6. He L, Hannon GJ. MicroRNAs: small RNAs with a big role in gene regulation. Nat Rev Genet. 2004;5(7):522-31.

7. Esquela-Kerscher A, Slack FJ. Oncomirs-microRNAs with a role in cancer. Nat Rev Cancer. 2006;6(4):259-69.

8. Calin GA, Croce CM. MicroRNA signatures in human cancers. Nat Rev Cancer. 2006;6(11):857-66.

9. Calin GA, Dumitru CD, Shimizu M, Bichi R, Zupo S, Noch E, et al. Frequent deletions and down-regulation of micro-RNA genes miR15 and miR16 at $13 q 14$ in chronic lymphocytic leukemia. Proc Natl Acad Sci U S A. 2002;99(24):15524-9.

10. Aqeilan Rl, Calin GA, Croce CM. miR-15a and miR-16-1 in cancer: discovery, function and future perspectives. Cell Death Differ. 2010;17(2):215-20.

11. Cimmino A, Calin GA, Fabbri M, lorio MV, Ferracin M, Shimizu M, et al. miR-15 and miR-16 induce apoptosis by targeting BCL2. Proceedings of the National Academy of Sciences of the United States of America. 2005;102(39):13944-9.

12. Linsley PS, Schelter J, Burchard J, Kibukawa M, Martin MM, Bartz SR, et al. Transcripts targeted by the microRNA-16 family cooperatively regulate cell cycle progression. Mol Cell Biol. 2007;27(6):2240-52.

13. Liu Q, Fu H, Sun F, Zhang H, Tie Y, Zhu J, et al. miR-16 family induces cell cycle arrest by regulating multiple cell cycle genes. Nucleic Acids Res. 2008:36(16):5391-404.

14. Bonci D, Coppola V, Musumeci M, Addario A, Giuffrida R, Memeo L, et al. The miR-15a-miR-16-1 cluster controls prostate cancer by targeting multiple oncogenic activities. Nat Med. 2008;14(11):1271-7.

15. Calin GA, Ferracin M, Cimmino A, Di Leva G, Shimizu M, Wojcik SE, et al. A MicroRNA signature associated with prognosis and progression in chronic lymphocytic leukemia. N Engl J Med. 2005;353(17):1793-801.

16. Bandi N, Zbinden S, Gugger M, Arnold M, Kocher V, Hasan L, et al. miR-15a and miR-16 are implicated in cell cycle regulation in a Rb-dependent manner and are frequently deleted or down-regulated in non-small cell lung cancer. Cancer Res. 2009;69(13):5553-9.

17. Chen X, Guo X, Zhang H, Xiang Y, Chen J, Yin Y, et al. Role of miR-143 targeting KRAS in colorectal tumorigenesis. Oncogene. 2009;28(10):1385-92.

18. Hsu SD, Tseng YT, Shrestha S, Lin YL, Khaleel A, Chou CH, et al. miRTarBase update 2014: an information resource for experimentally validated miRNA-target interactions. Nucleic acids research. 2014:42(Database issue):D78-85.

19. Altermann E, Klaenhammer TR: PathwayVoyager: pathway mapping using the Kyoto Encyclopedia of Genes and Genomes (KEGG) database. Bmc Genomics. 2005;6:60.

20. Tabas-Madrid D, Nogales-Cadenas R, Pascual-Montano A. GeneCodis3: a non-redundant and modular enrichment analysis tool for functional genomics. Nucleic acids research. 2012;40(Web Server issue):W478-483. 
21. Lewis BP, Shih IH, Jones-Rhoades MW, Bartel DP, Burge CB. Prediction of mammalian microRNA targets. Cell. 2003;115(7):787-98.

22. John B, Enright AJ, Aravin A, Tuschl T, Sander C, Marks DS. Human MicroRNA targets. PLoS Biol. 2004;2(11):e363.

23. Krek A, Grun D, Poy MN, Wolf R, Rosenberg L, Epstein EJ, et al.

Combinatorial microRNA target predictions. Nat Genet. 2005;37(5):495-500.

Submit your next manuscript to BioMed Central and take full advantage of:

- Convenient online submission

- Thorough peer review

- No space constraints or color figure charges

- Immediate publication on acceptance

- Inclusion in PubMed, CAS, Scopus and Google Scholar

- Research which is freely available for redistribution 